\title{
The Application of Image Processing Techniques in Analysis of Cigarette Packets Surface Defects
}

\author{
Yongdong QU ${ }^{1, \text { a }}$, Banggui HE ${ }^{1,2, b}$, Geye LIU $^{3, b}$ \\ ${ }^{1}$ Kunming University of Science and Technology, Kunming, 650500, China \\ ${ }^{2}$ Yunnan Packaging and Printing Engineering Technology Center, Kunming, 650500, China \\ ${ }^{3}$ Hongyun Honghe Group Qujing cigarette factory, Qujing, 655000, China \\ azdyf05@163.com, bynkmhe@163.com
}

\begin{abstract}
In the work of the GDX2 packaging units, commonly appear the defects of BOPP heat-sealing face of the hard small box such as relaxation, wrinkles, bubbles etc. So analyzing the heat sealing defects and has become the primary task to improve the GDX2 packaging units quality. This article based on the Matlab image processing technologies offers image recognition of the defects, and extracts the defects information of the image feature. After statistical analyses of the defect information extracted, the location of the defect concentrating region and the area of the defect region can be determined, and the research shows that the result is basically consistent with the actual distribution of the defects of the side of the hardware package of the small box. By using the image processing technology, the extraction of feature information on the heat sealing defects will be more accurate, and the data information collection of the defect characteristics provides the theoretical basis for analyzing the causes why the defects occur and improving the heat sealing system of the GDX2 packaging machine.
\end{abstract}

Index Terms - Image processing, MATLAB, Surface defect, Feature extraction

\section{Introduction}

The outside packaging of the product plays a vital role in enhancing the added value of products, enhancing the competitiveness in the market, and exploring markets. The $\mathrm{CH}$ small box packing machine in GDX2 packaging unit was designed with deficiencies in heat-sealing system, which leads to different degree of defects of the side-sealing film in the small hard packet, such as relaxation, wrinkles, bubbles and seriously affects the appearance quality of the products and the heat-sealing quality of the BOPP film[1]. For the small hard packet GDX2 packaging unit uses "U"-shaped film, $\mathrm{CH}$ small box packing machine wraps the packet with BOPP film around the packet, and seals the side of the packet under high temperature conditions by a soldering iron, so the packet has the packaging defects such as wrinkles, bubbles concentrated in the heat-sealing face. The manufacturers with the GDX2 packaging units have conducted various researches about the defects, but the side defects of the small hard packet still plague users in varying degrees[2], so analyses about the heatsealing defects of the side of the small hard packet have become particularly important. This article will transform the superficial information of the side of the packet into digital image information, with Matlab image processing Toolbox disposing the image information of the side of the packet, segmenting defects feature and extracting feature information.
Analyses of the defect information will supply theoretical bases and information features for improving the GDX2 units.

\section{Image Capture}

The features of the defects of the side heat-sealing face of the small packet mainly include film relaxation and others such as air bubbles, wrinkles emerging in the process of heat seal, which lead to uneven surface. When parallel light from the side irradiates the heat-sealing face, light diffuse reflection and refractive index difference among the air bubbles, wrinkles and flat surface will cause that the luminance in defects' areas and normal areas will be different. After the collection of different samples by using CCD camera, their luminance differences can be obtained by image processing, data processing calculating the information of the location and quantity of the defects.

Randomly remove 20 products in the normal work state of GDX2 packaging unit as a sample, the samples is grouped into each five a group. The samples heat sealing surface levels up, placed in a holder with the parallel light from the side irradiating the sample surface. By using the CCD camera Fixed on the holder, the pictures were taken on the front of the sample. The image of the side of the small packet is converted into digital image information, and the image of the side face of the small hard paket will be cut out after removing unrelated background information, as shown in Fig 1.

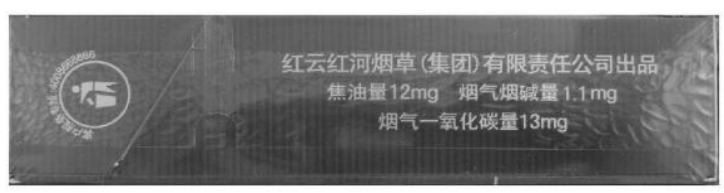

Fig 1 The side heat-sealing face

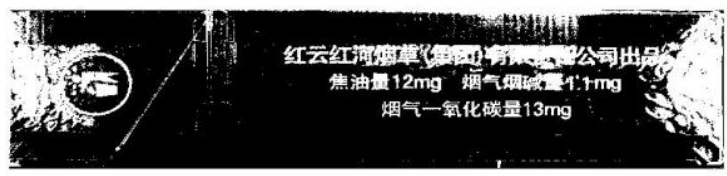

Fig 2 Segmentation of Gray level threshold value 


\section{Image Processing}

One of the original image is shown in Fig 1, which contains the feature information of heat-sealing defects and the background information of the hard packet. In order to extract feature information, firstly handle the original image and read it into the Matlab software, then removes interference and noise as well as differences with the image processing toolbox. So the original image could have obvious characteristics and is suitable for using a computer extract the feature information.

\section{Image Filter}

Due to the interference of the noise in the process of capturing images, image filtering can help reducing the effects of the noise. In the work, the median value filter, one of the nonlinear filtering technology, which could effectively restrain images noise and improve $\mathrm{S} / \mathrm{N}$, is used to remove the noise interference and in the mean time the image edges can be protected[3]. As a part of neighborhood operation, the median value filter is similar to convolution, but not weighted sum calculation. It sorts the neighborhood pixel according to the gray level, and then choose the median of the group as an output pixel value. The basic principle of the median value filter is that the value of the point of the digital image or sequence of numbers is replaced with the average value of all the neighborhood points. Assume that there is a onedimensional sequence. $f_{1,} f_{2, \ldots} f_{n}, m$ is said to be the length of the window.

The sequence is calculated with the median value filter operation. firstly extract $\mathrm{m}$ number from the input sequence, $f_{i-v, \ldots,} f_{i-1, \ldots,} f_{1, \ldots,} f_{i+1, \ldots,} f_{i+v}$ in the sequence, $i$ is said to be the center of the window, $v=\frac{m-1}{2}$, Then arrange these $\mathrm{m}$ points according to their numerical size, and select the serial number in the middle as the output value. This process is expressed as a mathematical formula:

$$
Y_{i}=\operatorname{Med}\left\{f_{i-v}, \cdots, f_{i}, \cdots, f_{i+v}\right\} \quad i \in Z, v=\frac{m-1}{2}
$$

For the sequence of two-dimensional median filter, the filter window is a two-dimensional window, which contains various shapes, such as linear, square, circle, cross, circular, etc.

Median filter of the two-dimensional data can be expressed as:

$$
Y_{i, j}=M \underset{A}{\operatorname{ed}}\left\{X_{i j}\right\} A \text { is said to be a filtering window. }
$$

The original image is handled by the median filter, the filter window is taken as $3 \times 3$ square window.

\section{Image Segmentation}

Image threshold segmentation is a more commonly used method to segment images, and it is especially suitable for coping with the target and background when they occupy different gray-scale range in the image. And it is a necessary image pre-processing process finished before image analysis, feature extraction and pattern recognition[4]. Threshold segmentation method is one of region-based image segmentation techniques, whose basic principle is dividing the image pixels into two categories by setting the different characteristics of the threshold. Assume that $f(x, y)$ is said to be the original image, and some kind of eigenvalue can be found in $f(x, y)$ according to a certain criteria, and it is the threshold value $\mathrm{T}$ that can split the image. So the image is divided into two parts, which is shown as follows:

$$
g(x, y)= \begin{cases}b_{0} & f(x, y)<T \\ b_{1} & f(x, y) \geq T\end{cases}
$$

Threshold segmentation needs to select a reasonable threshold to determine whether each pixel point in the image belongs to the target area $\mathrm{b}=1$, or the background region $\mathrm{b}=$ 0 , and accordingly generate the corresponding binary image. In order to highlight the required feature information of the defects and remove the doping background information, according to the rusults of the reseach, 0.45 is selected to split the original image as a gray scale threshold. The binarization of image is shown in Fig 2.

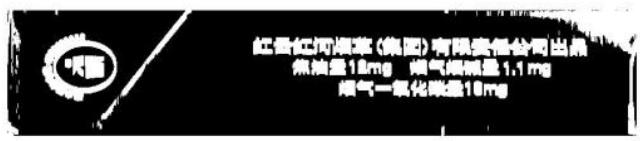

Fig 3 The background image after the expansion

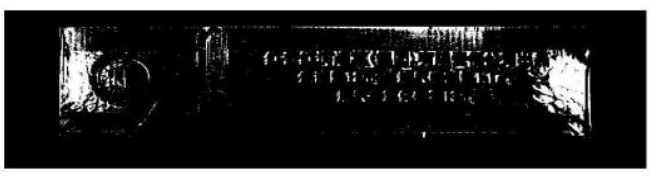

Fig 4 After deducting background information

\section{Background Subtraction}

For the most of the background information has been removed from the image segmented, the range of the gray value between the printed text and the defects characteristics are so similar that the printed characters threshold-segmented are still residual. Because the characters will affect the extraction and analyses of the defects feature, the image need to deduct the characters background information with differential operator. So firstly set the image on the side of the packet without BOPP as the background image, after threshold segmentation, the background image keeps only the characters information, then the characters background is handled by the image morphological operations, finally put the background image Fig 3 expanded subtract the image 2 by differential operator with the Matlab software image subtraction function[5]: 


\section{$\mathrm{Z}=\mathrm{imsubtract}(\mathrm{X}, \mathrm{Y})$}

The image after deducting the background which is obtained by image 2 subtract image 3 shown in Fig 4 .

\section{Feature Extraction}

After the process of background subtraction, only the BOPP film the bubbles and fold characteristics is left on the image, which is shown in Fig4. Then read the image into the Matlab Image Processing Toolbox and extract and fill out the image feature with the use of the related function. Firstly remove those too small dots that can be determined is not a bubble or fold, then mark the connected region to help filling out the number and areas of the bubbles, count the number and areas of the bubbles of the defects region[6]. Finally calculate the actual area of the bubbles according to the actual size of the hard packet and the pixel size, and list the defects characteristic of all samples. The result is shown in Table 1.

Table 1 The number and area of the bubbles

\begin{tabular}{cccc}
\hline Sample & $\begin{array}{c}\text { bubbles } \\
\text { numbers }\end{array}$ & $\begin{array}{c}\text { bubbles area } \\
\text { (pixel value) }\end{array}$ & $\begin{array}{c}\text { the actual area of } \\
\text { the bubbles }\left(\mathrm{mm}^{2}\right)\end{array}$ \\
\hline 1 & 95 & 115675 & 303 \\
2 & 92 & 120361 & 315 \\
3 & 96 & 92690 & 243 \\
4 & 111 & 126790 & 332 \\
5 & 65 & 89104 & 233 \\
6 & 84 & 91555 & 240 \\
7 & 78 & 100010 & 262 \\
8 & 83 & 97495 & 255 \\
9 & 86 & 103262 & 270 \\
10 & 61 & 95400 & 250 \\
11 & 141 & 104860 & 275 \\
12 & 99 & 98420 & 258 \\
13 & 100 & 96495 & 253 \\
14 & 97 & 99170 & 260 \\
15 & 91 & 92070 & 241 \\
16 & 92 & 97690 & 256 \\
17 & 125 & 131360 & 344 \\
18 & 115 & 137890 & 361 \\
19 & 114 & 152495 & 399 \\
20 & 96 & 124290 & 325 \\
Mean & 96 & 108354 & 284 \\
\hline
\end{tabular}

Determine the location of the defects characteristics, and extract the center of mass of bubbles the center of all bubbles in each sample, the centroid is calculated as follows [7]:

$$
\begin{aligned}
\bar{x}= & \frac{\iint_{D} x \rho(x, y) d \sigma}{\iint_{D} \rho(x, y) d \sigma}=\frac{\iint_{D} x \rho(x, y) d \sigma}{M} \\
\bar{y}= & \frac{\iint_{D} y \rho(x, y) d \sigma}{\iint_{D} \rho(x, y) d \sigma}=\frac{\iint_{D} y \rho(x, y) d \sigma}{M}
\end{aligned}
$$

For the area density is uniform, so the formulas can be simplified to:

$$
\bar{x}=\frac{\iint_{D} x d \sigma}{S}, \bar{y}=\frac{\iint_{D} y d \sigma}{S}
$$

The centers of the bubbles on all samples are drawn in the position scatter plot, which is shown in Fig 5.

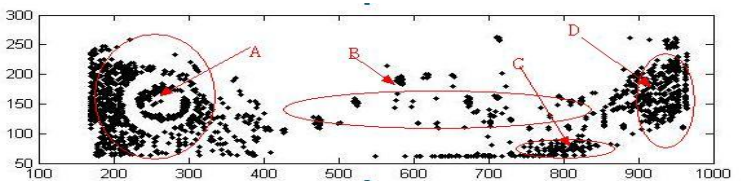

Fig 5 Scatter plot of the centroids location of the defect points

\section{Discussions}

With Matlab, The image of the heat-sealing BOPP film on the side of the hard packet is shown as Fig 4, which shows that the heat-sealing defects mainly include the fold in the in the folding process and the bubbles in the ironing process by the iron. Mark the defects regions in the image and fill out the information of the defects characteristics. By filling out the 20 samples, the largest number of the bubbles is 141, and the least number is 61 , and the average is 96 . The pixels maximum of the defects regions is 152495 and the actual area is $399 \mathrm{~mm}^{2}$. Of the whole samples, the minimum of the defects regionsis 89104 pixels which is calculated to be $233 \mathrm{~mm}^{2}$, and the average of the samples is 108354 which is calculated to be $284 \mathrm{~mm}^{2}$. The center of the image can be extracted with the Matlab image processing toolbox, and the centroid positions of the samples is painted in one coordinate system. The scatter plot of the defect points is shown as Fig 5, which clearly reflects the law of the distribution of bubbles positions.

(1) The heat-sealing defects of the BOPP film of the side of hard packet are shown in Fig 1, which mainly caused by the difference of the light transmittance caused by the bubbles, folds, etc. The defects feature information, as shown in Fig 2, is split by the image processing technology, and the result shows that this technology is more accurate than the previous eye-detection and able to convert the defect characteristics into data information.

(2) By observing the hard packet, the heat-sealing defects regions mainly concentrated at both ends and the middle of BOPP film. By observing the Fig 5, the actual locations of the defects regions are consistent with the data information.

(3) According to the statistics of the experiment, the datas of the defects feature of the samples is shown in table 1, and the location of centroids of the defects characteristics is shown as Fig 5, namely the scatter plot. By observing the Fig 5, the defects are mainly located in four regions. The region $\mathrm{A}$, region $\mathrm{B}$, region $\mathrm{C}$ and the region $\mathrm{D}$. However the region $\mathrm{C}$ is not a defect concentrated region but the deformation of the packet that results in the light reflection.

(4) Analyze the reason why the defects-concentrated area, as shown in Fig 5, is located in the regional A and D. The defects showed a triangular distribution near both ends of the hard packet, by contrasting the heat-sealing craft of the $\mathrm{CH}$ 
packaging machine, presumably the defects come due to the folding craft of folding BOPP film on the end face of the hard packet; By analyzing the region B where the BOPP film is heat-sealed on the side of the hard packet, the defects concentrated may be caused by the iron heat-sealing process parameters or the unreasonable design of the iron.

\section{Conclusions}

Extract and analyse the defects characteristics of the heatsealing BOPP film of the small hard packet, and locate the geometric features and the location characteristics of the defects. The experiment shows that this method can not only improve the speed of detecting the defects of the small hard packet and the accuracy of locating the defects, but also help finding the defects reason to, so the method can help to solve the heat-sealing defects of the GDX2 units fundamentally. There are some subtle characteristics of the defects cannot reach ideal recogniting results in the process of recogniting the defects feature, but the image processing technology used in the analyses of the defects is still worthy of deeply exploring for researchers.

\section{References}

[1] Xiangwei Wang and Cheren Chi. Packaging Engineering, 2008, 29 (7) P56-58.

[2] Shiqian Dong. Tobacco Science and Technology, 2001, (2) P22-23.

[3] Hongqi Sun, Weiying Shi and Yongfeng Ju, in: Image processing with medium value filter, volume 23(2) of Journal of Chang'an University (2003)P104.

[4] J.Astola and P. Kuosmanen. Fundamentals of Nonlinear Digital Filteirng. New York: C RC, 1997.5 (5) P56-62.

[5] Li Li, Lijun Sun, Chang Cheng. Journal of Tongji University,2011,39(5)P690-692.

[6] Chengming Wang, Yunhui Yan, Yingli Han. Cmputer Engineering and Applications, 2007, 43(13) P207.

[7] Abdel-Qader I,Abudayyeh O,Kelly M E,Analysis of edge detection techniques for crack identification in bridges. Journal of Computing in Civil Engineering, 2003,17(4):P255-263. 